\title{
Micromachining with square-shaped 1 ns-long pulses from an all-fiber Yb-doped laser-amplifier system
}

\author{
Kıvanç Özgören ${ }^{1}$, Bülent Öktem ${ }^{1}, F$. Ömer Ilday ${ }^{2}$, Ece Pasin $^{3}$, Koray Eken ${ }^{3}$ \\ ${ }^{I}$ Material Science and Nanotechnology Graduate Program, Bilkent University, 06800 Ankara, Turkey \\ ${ }^{2}$ Department of Physics, Bilkent University, 06800 Ankara, Turkey \\ ${ }^{3}$ FiberLAST, Ltd., 06531, Ankara, Turkey
}

\begin{abstract}
We demonstrate micromachining with 1ns-long pulses from an all-fiber laser. Fiber lasers generating uncompressible long pulses have been ignored as undesired operational modes, however their robust, low-repetition-rate operation is well suited to micromachining.

(C)2011 Optical Society of America

OCIS codes: (140.4050) Mode-locked lasers;(140.3390) Laser materials processing; (060.2320) Fiber optics amplifiers and oscillators
\end{abstract}

Lasers producing high-power, short pulses are increasingly being used for material processing applications, from micromachining to surface texturing and even tissues processing. Fiber lasers offer significant advantages with their environmentally robust operation, low-cost and compact size. The dynamics of fiber oscillators are governed by a rich interplay of Kerr nonlinearity, dispersion, and gain [1]. Over the past decade, a variety of mode-locking regimes have been identified, namely, the soliton, stretched-pulse, all-normal-dispersion, similariton and recently the soliton-similariton regimes. A commonly encountered, but usually ignored mode is when the laser produces stable, but long pulses, which cannot be compressed to the transform limit. This mode of operation is referred to as noise-like operation [2] or square-pulse generation [3], which are obtained from net anomalous-dispersion and net normal-dispersion cavities, respectively. An interesting prospect is to amplify the square-shaped pulses for use in material processing: the oscillator naturally operates at low repetition rates, eliminating the need for pulse picking and produces $\sim 1$ ns-longpulses. This is an interesting temporal regime, where plasma formation is sustained long enough for efficient ablation, but short enough to limit thermal effects to surrounding areas. Even if the optical phase oscillates randomly under the pulse envelope, we expect this to be of no consequence for material processing.

Here, we report on the development of an all-fiber-integrated oscillator utilizing a nonlinear optical loop mirror (NOLM) [4] generating 1 ns-long pulses. The pulse-to-pulse fluctuations of this regime are characterized for the first time. Following fiber amplification, these pulses are used in micromachining titanium surfaces and compared to a picosecond and a nanosecond laser.. Thus, the entire setup is robust and misalignment-free since only fiberintegrated components are used both in the oscillator and the amplifier (Fig. 1).

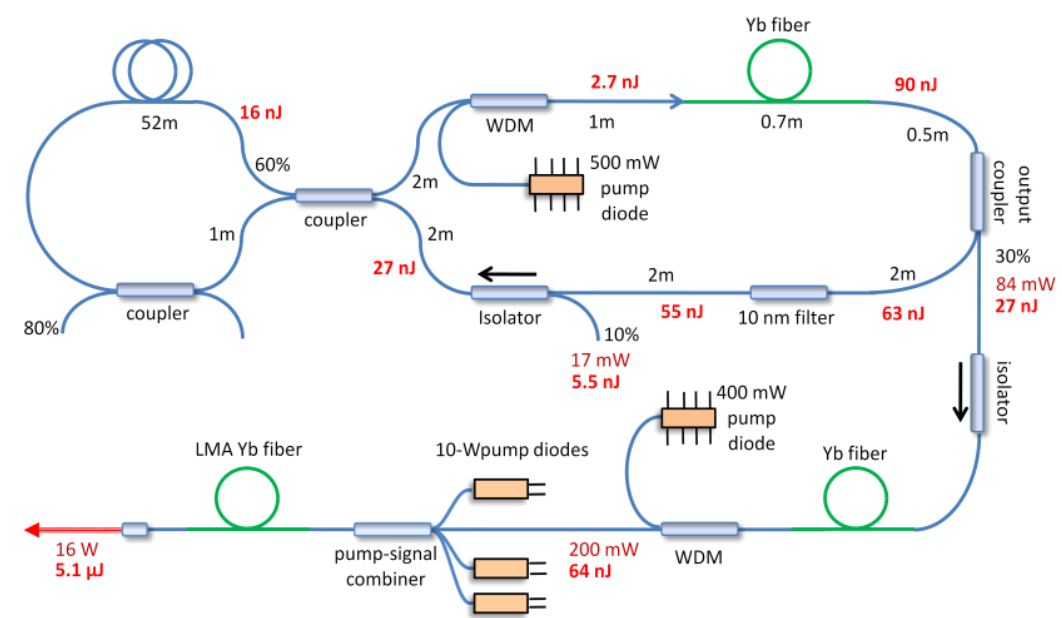

Fig. 1. Schematic diagram of the oscillator-amplifier setup. WDM: wavelength-division multiplexer; LMA: large mode area. The fiber lengths, powers and pulse energies are indicated. 
The cavity comprises of a $0.7 \mathrm{~m}$-long segment of Yb-doped fiber, followed by $30 \%$ output coupler, a 10 -nm bandpass filter, an inline isolator to ensure and the NOLM segment. The gain fiber is pumped with up to $500 \mathrm{~mW}$ of power at $980 \mathrm{~nm}$. The NOLM consists of a 40\%-60\% input coupler and a second coupler with $20 \%-80 \%$ splitting ratio. The fiber loop hasa $50 \mathrm{~m}$-long HI-1060 fiber segment. At full pump power, the pulse energy is $90 \mathrm{~nJ}$ after the gain fiber. The pulse energy entering the NOLMis $\sim 27 \mathrm{~nJ}$. We estimate that the pulses accumulate a relative phase shift of $\sim \pi$ in the Sagnac loop, which maximizes its transmittance. Fig. 2(a) shows the optical spectrum recorded from the $10 \%$ tap port of the isolator. Fig. 2(b) shows the temporal profile of the pulses as measured with a $50-\mathrm{GHz}$ sampling oscilloscope and 12-GHz photodiode, with a combined rise time of $\sim 30 \mathrm{ps}$. The pulse width is slightly less than $1 \mathrm{~ns}$. The RF spectrum is found to be clean of modulations down to $-70 \mathrm{dBc}(\mathrm{Fig}$. 3(c, d)). The short-term power stability of the pulse train is characterized by its relative intensity noise (RIN) obtained using the standard method [5]. The integrated noise over the range $3 \mathrm{~Hz}-250 \mathrm{kHz}$ corresponds to pulse-to-pulse fluctuations of $0.6 \%$. The pulse train generated by the oscillator seeds the two-stage fiber amplifier, which is similar to the system described in [6]. A preamplifier is followed by a large-mode area fiber amplifier cladding-pumped by three fibercoupled 10-W multi-mode pump diodes through a signal-pump combiner. The maximum output power is $16 \mathrm{~W}$, corresponding to $5.14 \mu \mathrm{J}$ of pulse energy and $5.1 \mathrm{~kW}$ of peak power. The beam profile is Gaussian with $\mathrm{M}^{2}<1.2$.
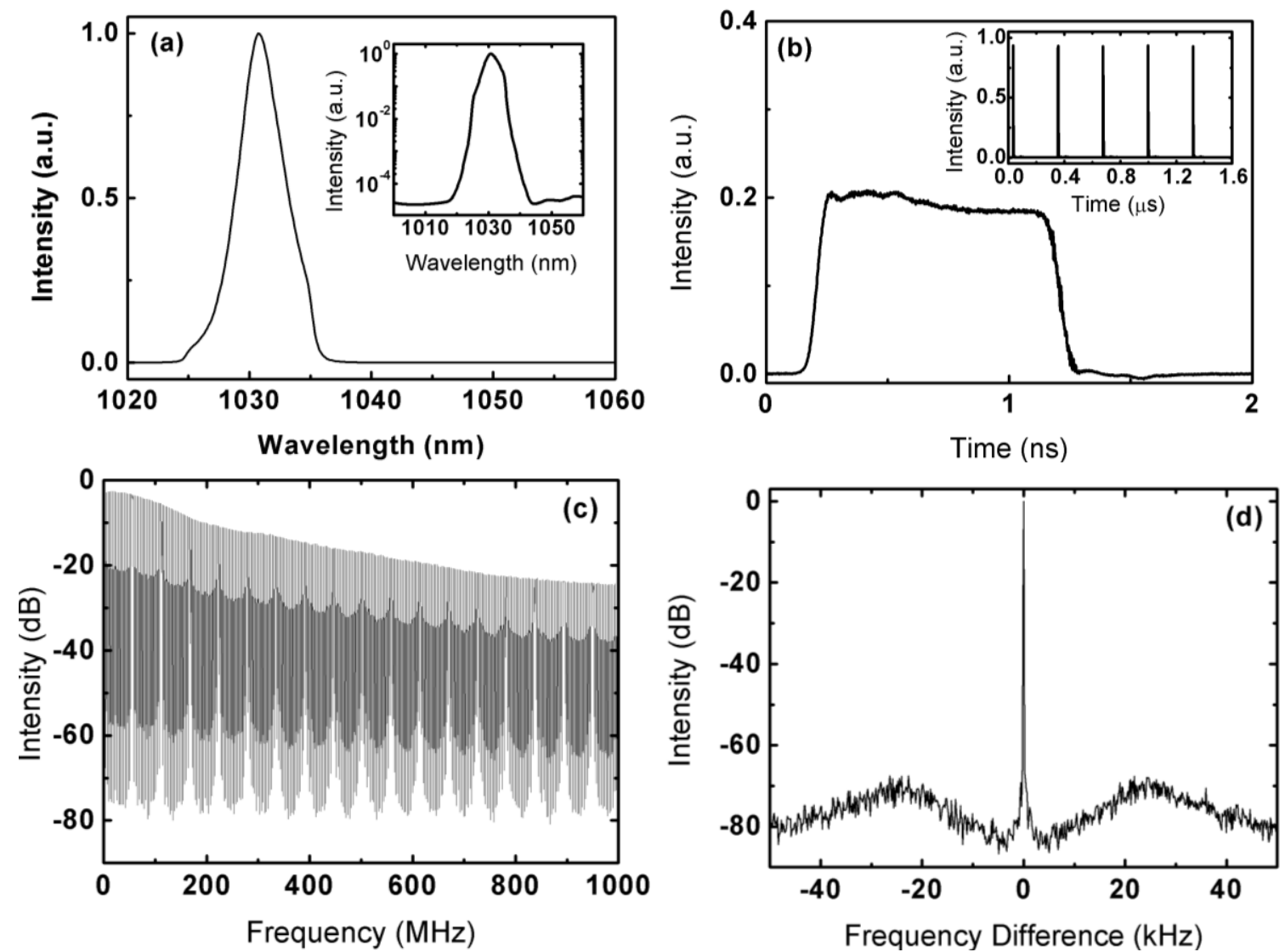

Fig. 3.(a) Optical spectrum of the pulse train measured at the $10 \%$ output port. (b) Pulse shape measured with a 30 ps-rise time sampling scope. Inset: Pulse train measured with a $1 \mathrm{GHz}$-bandwidth real-time oscilloscope.(c) RF spectrum of the pulse train. (d) RF spectrum of an individual comb line demonstrating low-noise operation.

In order to test the utility of this system in material processing, we use a galvo scanner, followed by an f-theta objective, which focuses the beam to a spot size of $20 \mu \mathrm{m}$. As target, we use polished titanium surfaces. It is found that for this target approximately $2 \mathrm{~W}$ of power is sufficient. For comparison, we use an industrial fiber laser producing 70 ns-long pulses and a home-built fiber chirped pulse amplifier (CPA). The fiber CPA system is operated without the final pulse compression stage and delivers 20 ps-long pulses at [7]. Fig. 4 shows scanning electron microscope (SEM) images of processed Ti surfacein comparison with the imagesfor $20 \mathrm{ps}$ and $70 \mathrm{~ns}$ pulses taken from [4].The heat-affected zone is small compared to 70-ns system, and essentially the same as with the 20-ps system.Fig. 5 shows close-in images of the patterns created. The holes have a diameter of $\sim 35 \mu \mathrm{m}$. The deposits 
near the holes are predominantly $\mathrm{TiO}_{2}$, as determined from EDX analysis and Raman spectroscopy. We also produced trenches on the titanium surface, scanning at a speed of $0.1 \mathrm{~mm} / \mathrm{s}$ at $0.75 \mathrm{~W}$ of average power. The depth of the trenches increases with increasing fluence.
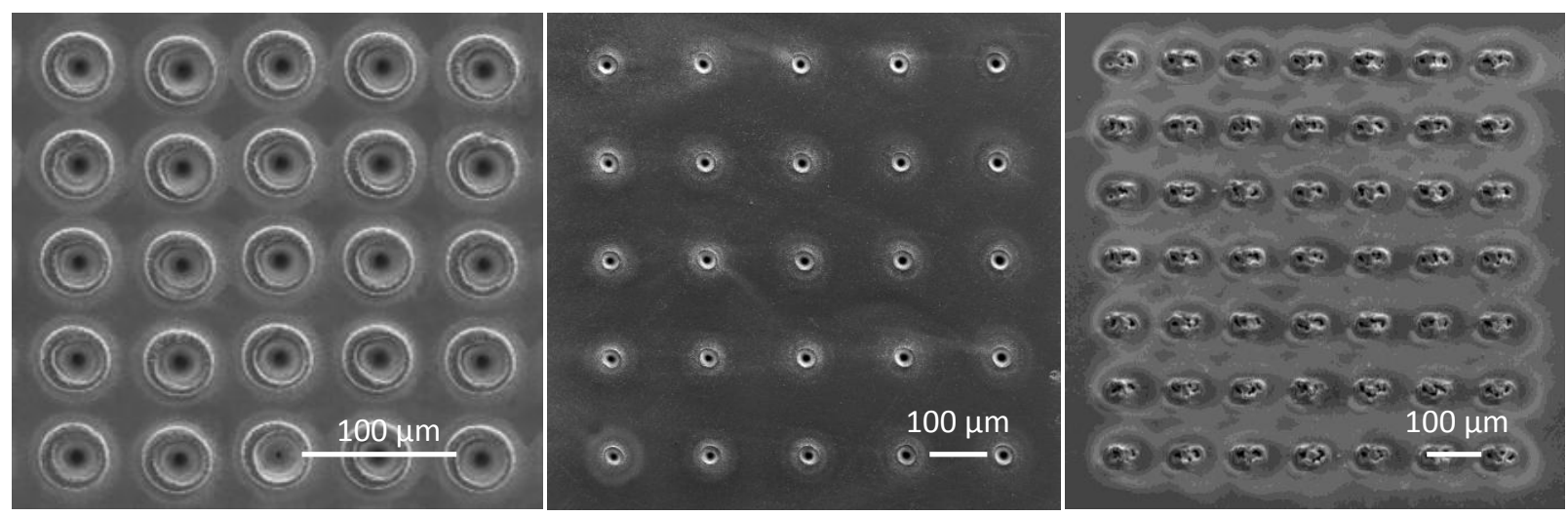

Fig. 4.Micromachined Ti surfacesusing (a) 20 ps-long, (b) 1 ns-long, and (c) 70 ns-long pulses.

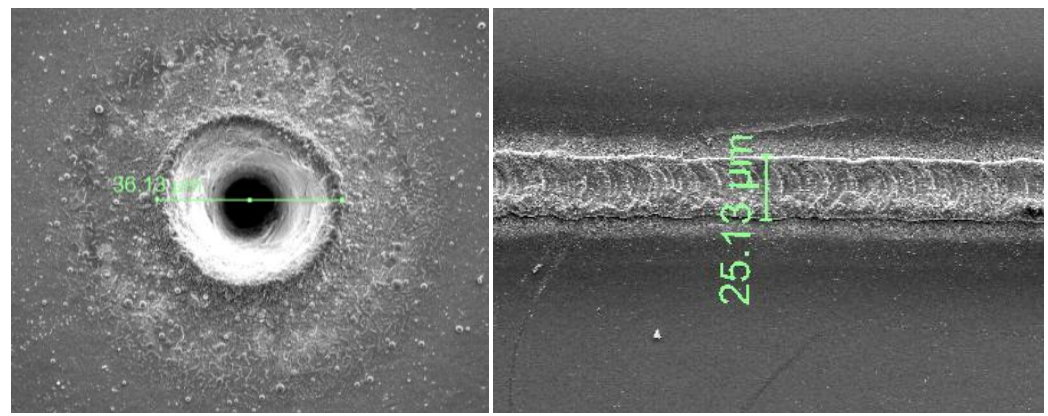

Fig. 5.(a) Close-up of a single hole with $\mathrm{TiO}_{2}$ deposits nearby,and (c) a line pattern created by the 1 ns-long square pulses. No post processing or surface cleaning has been performed.

In conclusion, we have demonstrated, for the first time to our knowledge, the use of 1 ns-long,uncompressible, square-pulses generated from an $\mathrm{Yb}$-fiber laser in precision micromachining. The simple all-fiber cavity design uses a NOLM to initiate pulse operation at the fundamental repetition rate of $3.1 \mathrm{MHz}$, eliminating the need for pulse picking. The laser has an integrated RIN of $0.6 \%$. We compare the performance of the laser with an industrial nanosecond fiber laser and a chirped-pulse fiber amplifier system producing 20 ps-long pulses. The quality and repeatability of micromachining is similar to those obtained with picosecond pulses and better than with the nanosecond pulsed lasers typically utilized in industrial micromachining. To date, this mode of operation of fiber lasers has been largely ignored as an undesirable mode of operation. In fact, for long cavities, this regime occupies a large portion of the phase space, exhibiting very high tolerance of environmental perturbations. As such we expect it to find wide use in industrial applications.

\section{References}

[1] H. A. Haus, “Mode-locking of lasers," IEEE J. Sel. Top. Quantum Electron. 6, 1173 (2000).

[2] M. Horowitz, Y. Barad, and Y. Silberberg, "Noiselike pulses with a broadband spectrum generated from an erbium-doped fiber laser," Opt. Lett. 22, 799-801 (1997).

[3] L. M. Zhao, D. Y. Tang, T. H. Cheng, and C. Lu, "Nanosecond square pulse generation in fiber lasers with normal dispersion," Opt. Comm. 272, 431 (2007).

[4] N. J. Doran and David Wood, "Nonlinear-optical loop mirror," Opt. Lett. 13, 56 (1988).

[5] R. P. Scott, C. Langrock, and B. H. Kolner, "High dynamic range laser amplitude and phase noise measurement techniques," IEEE J. Quantum Electron. 7, 641 (2001).

[6] P. K. Mukhopadhyay, K. Özgören, I. L. Budunoglu, and F. Ö. Ilday, "All-fiber low-noise high-power femtosecondYb-fiber amplifier system seeded by an all-normal dispersion fiber oscillator," IEEE J. Sel. Top. Quantum Electron.15, 145 (2009).

[7] B. Oktem, H. Kalaycioglu, M. Erdoğan, S. Yavaş, P. Mukhopadhyay, U. H. Tazebay, Y. Aykaç, K. Eken, and F. Ö. Ilday, "Surface texturing of dental implant surfaces with an ultrafast fiber laser," in Conference on Lasers and Electro-Optics, OSA Technical Digest (CD) (Optical Society of America, 2010), paper JTuD15. 